\title{
Multiple Solutions for an Equation of Kirchhoff Type: Theoretical and Numerical Aspects
}

\author{
A.L.M. MARTINEZ ${ }^{1}$, E.V. CASTELANI ${ }^{2}$, G.M. BRESSAN ${ }^{1}$ and E.W. STIEGELMEIER ${ }^{1}$ \\ Received on March 15, 2018 / Accepted on June 25, 2018
}

\begin{abstract}
A nonlinear boundary value problem related to an equation of Kirchhoff type is considered. The existence of multiple positive solutions is proved through Avery-Peterson Fixed Point Theorem. A numerical method based on Levenberg-Marquadt algorithm combined with a heuristic process is present in order to align numerical and theoretical aspects.
\end{abstract}

Keywords: Multiple solution, Kirchhoff Equation, numerical solutions.

\section{INTRODUCTION}

In this paper we present a study on second order equation Kirchhoff problem, given by

$$
\left\{\begin{array}{c}
M\left(\left\|u^{\prime}\right\|_{2}^{2}\right) u^{\prime \prime}+q(t) f\left(t, u, u^{\prime}\right)=0 \\
u(0)=0, u(1)=0
\end{array}\right.
$$

where $M: \mathbb{R} \rightarrow \mathbb{R}, f:[0,1] \times \mathbb{R} \times \mathbb{R} \rightarrow \mathbb{R}$ and $q: \mathbb{R}^{+} \rightarrow \mathbb{R}$ are continuous maps.

Variations of (1.1) can be related to stationary state of Kirchhoff equation [8]. For example, as elucidated in [11] considering argument reflection and later in [1] with more general boundary conditions a stationary state of the Kirchhoff equation of kind of:

$$
u_{t t}-\left[c_{0}+c_{1} \int_{0}^{L}\left|u_{x}\right|^{2} d x\right] u_{x x}=0,
$$

can be associated with their respectives equations. This kind of equation commonly appear in the context of free vibrations in elastic strings, consequently, they form a relevant object of study.

Many authors have studied problems related to (1.2). For a wide spectrum of study, we recommend [2], [3], [6], [10] and more recently [5]. Concerning the problem (1.1) we can observe

\footnotetext{
*Corresponding author: André Luís Machado Martinez - E-mail: andrelmmartinez@yahoo.com.br

${ }^{1}$ Departamento Acadêmico de Matemática, Universidade Tecnológica Federal do Paraná, Cornélio Procópio, PR, Brasil. E-mail: andrelmmartinez@yahoo.com.br, galbressan@gmail.com, elenicew@utfpr.edu.br

2 Departamento de Matemática, DMA, UEM - Universidade Estadual de Maringá, Av. Colombo, 5790, 87020-900, Maringá, PR, Brasil. E-mail: evcastelani@uem.br
} 
by [12] that studies with theoretical aspects were developed using Banach's Fixed Point Theorem or Leray-Schauder Alternative combined with Krasnoselskii's Theorem. Consequently, it is natural to ask about existence results by using Avery-Peterson Theorem [4]. In this sense, this work complement previous results of existence with the cited theorem, and this study is present in Section 2.

Numerical studies related to second order equations, normally, are presented as illustration of the existence Banach's Fixed Point Theorem applied to equation but no strategy is given to ilustraste more general results (like the existence results provided by Avery-Peterson Theorem). The common sense suggests that optimization methods combined with heuristic processes can provide good results to find multiple numerical solutions. As consequence, a method based on Levenberg-Maquardt [13,9] is shown in Section 3 and comparisons with other methods using classical strategies are established. Still, in Section 3, a simple but effective heuristic process is introduced in order to find multiple numerical solutions. Final remarks are given in Section 4.

\section{MULTIPLE SOLUTIONS}

Let $C^{1}[0,1]$ be the Banach space of the continuously differentiable functions in $[0,1]$. Let us consider $E=\left\{u \in C^{1}[0,1] ; u(0)=u(1)=0\right\}$ with the norm

$$
\|u\|_{E}=\left\|u^{\prime}\right\|_{\infty}
$$

We begin this section by observing that the solutions of (1.1) can be written as:

$$
u(t)=\int_{0}^{1} G(t, s) q(s) \frac{f\left(s, u(s), u^{\prime}(s)\right)}{M\left(\left\|u^{\prime}\right\|_{2}^{2}\right)} d s
$$

where $G$ is the Green's function

$$
G(t, s)= \begin{cases}s(1-t), & s \leq t \\ t(1-s), & t \leq s\end{cases}
$$

Considering the result that we will show, we must note some properties of the function $G$. In fact, we have that

$$
\partial_{t} G(t, s)= \begin{cases}-s, & s \leq t \\ 1-s, & t \leq s\end{cases}
$$

then $G$ satisfies:

$$
G(t, s)=|G(t, s)| \leq\left|\partial_{t} G(t, s)\right|
$$

Let $\bar{m}$ be a constant in $[0,1 / 2]$. Thus, we can obtain the inequalities

$$
G(t, s) \geq \bar{m} G(s, s), \forall x \in[\bar{m}, 1-\bar{m}]
$$

and

$$
G(t, s) \leq G(s, s), \forall t \in[0,1] .
$$


Thus, concerning the representation given in (2.1) we define the operator $T: E \rightarrow E$ as:

$$
T(u)=\int_{0}^{1} G(t, s) q(s) \frac{f\left(s, u(s), u^{\prime}(s)\right)}{M\left(\left\|u^{\prime}\right\|_{2}^{2}\right)} d s,
$$

where $M$ and $f$ are assumed to be continuous functions and there is a constant $M_{0}>0$ such that $M(y) \geq M_{0}$ for all $y$ in the domain of $M$.

We claim that $T$ is continuous and completely continuous. Continuity follows immediately from the Lebesgue dominated convergence theorem and the fact that

$$
\begin{aligned}
\left|T(u)(t)-T\left(u_{n}\right)(t)\right| & \leq \int_{0}^{1} G(t, s) q(s)\left|\frac{f\left(s, u(s), u^{\prime}(s)\right)}{M\left(\left\|u^{\prime}\right\|_{2}^{2}\right)}-\frac{f\left(s, u_{n}(s), u_{n}^{\prime}(s)\right)}{M\left(\left\|u_{n}^{\prime}\right\|_{2}^{2}\right)}\right| d s \\
& \leq \int_{0}^{1} G(s, s) q(s)\left|\frac{f\left(s, u(s), u^{\prime}(s)\right)}{M\left(\left\|u^{\prime}\right\|_{2}^{2}\right)}-\frac{f\left(s, u_{n}(s), u_{n}^{\prime}(s)\right)}{M\left(\left\|u_{n}^{\prime}\right\|_{2}^{2}\right)}\right| d s \\
& \leq \int_{0}^{1} s(1-s) q(s)\left|\frac{f\left(s, u(s), u^{\prime}(s)\right)}{M\left(\left\|u^{\prime}\right\|_{2}^{2}\right)}-\frac{f\left(s, u_{n}(s), u_{n}^{\prime}(s)\right)}{M\left(\left\|u_{n}^{\prime}\right\|_{2}^{2}\right)}\right| d s
\end{aligned}
$$

with $u_{n}, u \in E$. To show complete continuity we will use the Arzela-Ascoli's theorem. Let $\Omega \subseteq E$ be bounded, in other words, there exists $\Lambda_{0}>0$ with $\|u\| \leq \Lambda_{0}$ for each $u \in \Omega$. Now if $u \in \Omega$ we have

$$
\left|(T u)^{\prime}(t)\right| \leq \int_{0}^{1}\left|G^{\prime}(t, s)\right| H_{\Lambda_{0}}(s) d s
$$

where $H_{\Lambda_{0}}$ is determined by the bounded set and functions $q, f$ and $M$. It is easy to check that $H_{\Lambda_{0}}(s) \in L^{1}[0,1]$. Then imply that $T \Omega$ is a bounded equicontinuous family on $[0,1]$. Consequently the Arzela-Ascoli theorem implies $T: E \rightarrow E$ is completely continuous.

Consider the following hypotheses:

(A1) There are positive constants $d, A, B$ such that:

- $q(t) f(t, u, v) \geq 0, \forall(t, u, v) \in[0,1] \times\left[-\frac{d}{2}, \frac{d}{2}\right] \times[-d, d]$

- $\forall(t, u, v) \in[0,1] \times\left[-\frac{d}{2}, \frac{d}{2}\right] \times[-d, d]$, then $|f(t, u, v)| \leq \frac{d A}{r_{1}}$, where

$$
r_{1}=\max _{t \in[0,1]}\left\{\int_{0}^{1}\left|\partial_{t} G(t, s) q(s)\right| d s\right\} ;
$$

- $A \leq M\left(\left\|u^{\prime}\right\|_{2}^{2}\right) \leq B, \forall\|u\|_{E} \leq d$

Next, we define a cone $P$ by

$$
P=\left\{u \in C^{1}[0,1] ; u \geq 0, u(0)=u(1)=0\right\} .
$$

Remark 1. Denoting

$$
F(t)=\int_{0}^{1} G(t, s) q(s) \frac{f\left(s, u(s), u^{\prime}(s)\right)}{M\left(\left\|u^{\prime}\right\|_{2}^{2}\right)} d s
$$


we can extract some properties related to F. Since

$$
F^{\prime \prime}(t)=-q(t) \frac{f\left(t, u(t), u^{\prime}(t)\right)}{M\left(\left\|u^{\prime}\right\|_{2}^{2}\right)} \leq 0,
$$

from $(A 1)$, we find that $F$ is concave, and since $F(0)=F(1)=0$, we can conclude that

$$
\begin{aligned}
\min _{t \in\left[\frac{1}{4}, \frac{3}{4}\right]} \int_{0}^{1} G(t, s) q(s) \frac{f\left(s, u(s), u^{\prime}(s)\right)}{M\left(\left\|u^{\prime}\right\|_{2}^{2}\right)} d s=\min \left\{\int_{0}^{1} G\left(\frac{1}{4}, s\right) q(s) \frac{f\left(s, u(s), u^{\prime}(s)\right)}{M\left(\left\|u^{\prime}\right\|_{2}^{2}\right)} d s,\right. \\
\left.\int_{0}^{1} G\left(\frac{3}{4}, s\right) q(s) \frac{f\left(s, u(s), u^{\prime}(s)\right)}{M\left(\left\|u^{\prime}\right\|_{2}^{2}\right)} d s\right\}
\end{aligned}
$$

For the purpose of this work we need to introduce the main tools.

\section{Avery-Peterson theorem.}

Now, we need to consider the convex sets

$$
\begin{gathered}
P(\gamma, d)=\{x \in P \mid \gamma(x)<d\} \\
P(\gamma, \alpha, b, d)=\{x \in P \mid b \leq \alpha(x) \text { and } \gamma(x)<d\} \\
P(\gamma, \theta, \alpha, b, c, d)=\{x \in P \mid b \leq \alpha(x), \theta(x) \leq c \text { and } \gamma(x)<d\}
\end{gathered}
$$

and the closed set

$$
R(\gamma, \psi, a, d)=\{x \in P \mid a \leq \psi(x) \text { and } \gamma(x)<d\}
$$

Theorem 2. Let $P$ be a cone in a real Banach space X. Let $\gamma$ and $\theta$ nonnegative continuous convex functionals on $P, \alpha$ be a nonnegative continuous concave functional on $P$, and $\psi$ be a nonnegative continuous functional on $P$ satisfying $\psi(\lambda x) \leq \lambda \psi(x)$ for $0 \leq \lambda \leq 1$, such that for some positive numbers $\mu$ and $d$,

$$
\alpha(x) \leq \psi(x) \text { and }\|x\| \leq \mu \gamma(x)
$$

for all $x \in \overline{P(\gamma, d)}$. Suppose

$$
T: \overline{P(\gamma, d)} \rightarrow \overline{P(\gamma, d)}
$$

is completely continuous and there exist positive numbers $a, b, c$ with $a<b$, such that

$$
\begin{gathered}
\{u \in P(\gamma, \theta, \alpha, b, c, d) \mid \alpha(u)>b\} \neq \emptyset \text { and } \\
u \in P(\gamma, \theta, \alpha, b, c, d) \Rightarrow \alpha(T u)>b \\
\alpha(T u)>b \text { for } u \in P(\gamma, \alpha, b, d) \text { with } \theta(T u)>c, \\
0 \notin R(\gamma, \psi, a, d) \text { and } \psi(T u)<a \text { for } \\
u \in R(\gamma, \psi, a, d) \text { with } \psi(u)=a .
\end{gathered}
$$


Then $T$ has at least three distinct fixed points in $\overline{P(\gamma, d)}$.

In our main result (given by Theorem 2) we will show that the Problem 1.1 has at least three positive solutions.

Theorem 3. Suppose that the hypothesis (A1) is satisfied. Suppose, in addition, that there exist $a, 0<a<d$ such that $f$ satisfies the following conditions:

$$
\begin{aligned}
& |f(t, u, v)|<\frac{A a}{r_{2}}, \forall(t, u, v) \in[0,1] \times[0, a] \times[-d, d], \\
& \text { where } r_{2}=\max _{t \in[0,1]}\left\{\int_{0}^{1} G(t, s)|q(s)| d t\right\} .
\end{aligned}
$$

$$
\begin{aligned}
& |f(t, u, v)|>\frac{2 a B}{r_{3}}, \forall(t, u, v) \in[0,1] \times[2 a, 8 \sqrt{2} a] \times[-d, d] \\
& \text { where } r_{3}=\min \left\{\int_{0}^{1} G\left(\frac{1}{4}, s\right)|q(s)| d s, \int_{0}^{1} G\left(\frac{3}{4}, s\right)|q(s)| d s\right\} .
\end{aligned}
$$

Then, Problem (1.1) has at least three positive solutions.

Proof. We will apply Avery-Peterson theorem (as stated in [4]). Let us consider $T$ and $P$ as defined before. Furthermore, we need define the following functionals

$$
\begin{aligned}
\gamma(u) & =\|u\|_{E}, \\
\psi(u) & =\max _{t \in[0,1]}|u(t)|, \\
\theta(u) & =\left[\int_{\frac{1}{4}}^{\frac{3}{4}}[u(t)]^{2} d t\right]^{\frac{1}{2}}, \\
\alpha(u) & =\min _{t \in\left[\frac{1}{4}, \frac{3}{4}\right]}|u(t)| .
\end{aligned}
$$

Therefore, we need to verify that

$$
T: \overline{P(\gamma, d)} \rightarrow \overline{P(\gamma, d)}
$$

is completely continuous and there exist positive numbers $b$ and $c$ with $a<b$, such that

$$
\begin{gathered}
\{u \in P(\gamma, \theta, \alpha, b, c, d) \mid \alpha(u)>b\} \neq \emptyset \text { and } \\
u \in P(\gamma, \theta, \alpha, b, c, d) \Rightarrow \alpha(T u)>b \\
\alpha(T u)>b \text { for } u \in P(\gamma, \alpha, b, d) \text { with } \theta(T u)>c, \\
0 \notin R(\gamma, \psi, a, d) \text { and } \psi(T u)<a \text { for } \\
u \in R(\gamma, \psi, a, d) \text { with } \psi(u)=a .
\end{gathered}
$$


Using $(A 1)$ we have $T u \geq 0$ if $\gamma(u)=\|u\|_{E} \leq d$, and obtain:

$$
\begin{aligned}
\|T u\|_{E} & =\left\|(T u)^{\prime}\right\|_{\infty} \\
& \leq \frac{d A}{r_{1}} \max _{t \in[0,1]} \int_{0}^{1} \frac{\left|\partial_{x} G(t, s) q(s)\right|}{M\left(\left\|u^{\prime}\right\|_{2}^{2}\right)} d s \\
& \leq d .
\end{aligned}
$$

Therefore $T$ applies $\overline{P(\gamma, d)}$ in $\overline{P(\gamma, d)}$.

Now, we consider

$$
b=2 a
$$

and

$$
c=8 \sqrt{2} a .
$$

Clearly, we have $\{u \in P(\gamma, \theta, \alpha, b, c, d) \mid \alpha(u)>b\} \neq \emptyset$. Let us demonstrate (2.8). Using (A3) we obtain

$$
\begin{aligned}
\alpha(T u) & =\min _{t \in\left[\frac{1}{4}, \frac{3}{4}\right]}(T u)(t) \\
& =\min _{t \in\left[\frac{1}{4}, \frac{3}{4}\right]}\left(\int_{0}^{1} G(t, s) q(s) \frac{f\left(s, u(s), u^{\prime}(s)\right)}{M\left(\left\|u^{\prime}\right\|_{2}^{2}\right)} d s\right) \\
& =\min _{x \in\left[\frac{1}{4}, \frac{3}{4}\right]} \int_{0}^{1} G(t, s)|q(s)| \frac{\left|f\left(s, u(s), u^{\prime}(s)\right)\right|}{M\left(\left\|u^{\prime}\right\|_{2}^{2}\right)} d s \\
& =\min \left\{\int_{0}^{1} G\left(\frac{1}{4}, s\right)|q(s)| \frac{\left|f\left(s, u(s), u^{\prime}(s)\right)\right|}{M\left(\left\|u^{\prime}\right\|_{2}^{2}\right)} d s, \int_{0}^{1} G\left(\frac{3}{4}, s\right)|q(s)| \frac{\left|f\left(s, u(s), u^{\prime}(s)\right)\right|}{M\left(\left\|u^{\prime}\right\|_{2}^{2}\right)} d s\right\} \\
& >\frac{2 a B}{r_{3}} \min \left\{\int_{0}^{1} G\left(\frac{1}{4}, s\right) \frac{|q(s)|}{M\left(\left\|u^{\prime}\right\|_{2}^{2}\right)} d s, \int_{0}^{1} G\left(\frac{3}{4}, s\right) \frac{|q(s)|}{M\left(\left\|u^{\prime}\right\|_{2}^{2}\right)} d s\right\} \\
& >\frac{2 a}{r_{3}} \min \left\{\int_{0}^{1} G\left(\frac{1}{4}, s\right)|q(s)| d s, \int_{0}^{1} G\left(\frac{3}{4}, s\right)|q(s)| d s\right\} \\
& \geq 2 a=b .
\end{aligned}
$$

Let us demonstrate (2.9). Let $u \in P(\gamma, \alpha, b, d)$ with $\theta(T u)>c$. Then 


$$
\begin{aligned}
\alpha(T u) & =\min _{t \in\left[\frac{1}{4}, \frac{3}{4}\right]}\left\{\int_{0}^{1} G(t, s) q(s) \frac{f\left(s, u(s), u^{\prime}(s)\right)}{M\left(\left\|u^{\prime}\right\|_{2}^{2}\right)} d s\right\} \\
& \geq \frac{1}{4}\left(\int_{0}^{1} G(t, s) q(s) \frac{f\left(s, u(s), u^{\prime}(s)\right)}{M\left(\left\|u^{\prime}\right\|_{2}^{2}\right)} d s\right) \\
& \geq \frac{1}{4} \max _{t \in[0,1]}\left\{\int_{0}^{1} G(t, s) q(s) \frac{f\left(s, u(s), u^{\prime}(s)\right)}{M\left(\left\|u^{\prime}\right\|_{2}^{2}\right)} d s\right\} \\
& \geq \frac{1}{4 \sqrt{2}} \theta\left(\int_{0}^{1} G(t, s) q(s) \frac{f\left(s, u(s), u^{\prime}(s)\right)}{M\left(\left\|u^{\prime}\right\|_{2}^{2}\right)} d s\right) \\
& \geq \frac{1}{4 \sqrt{2}} \theta(T u) \\
& >\frac{1}{4 \sqrt{2}} c=b .
\end{aligned}
$$

Now, let us show (2.10). Thus, let $u \in R(\gamma, \psi, a, d)$ with $\psi(u)=a$. From $(A 1)-(A 2)$ we have,

$$
\begin{aligned}
\psi(T u) & =\max _{t \in[0,1]}|T u(t)| \\
& \leq \max _{t \in[0,1]}\left(\int_{0}^{1} G(t, s) q(s) \frac{f\left(s, u(s), u^{\prime}(s)\right)}{M\left(\left\|u^{\prime}\right\|_{2}^{2}\right)} d s\right) \\
& \leq \max _{t \in[0,1]}\left(\int_{0}^{1} G(t, s)|q(s)| \frac{\left|f\left(s, u(s), u^{\prime}(s)\right)\right|}{M\left(\left\|u^{\prime}\right\|_{2}^{2}\right)} d s\right) \\
& \leq \frac{A a}{r_{2}} \max _{x \in[0,1]}\left(\int_{0}^{1} G(t, s) \frac{|q(s)|}{M\left(\left\|u^{\prime}\right\|_{2}^{2}\right)} d s\right) \\
& \leq \frac{a}{r_{2}} \max _{x \in[0,1]}\left(\int_{0}^{1} G(t, s)|q(s)| d s\right) \\
& \leq a .
\end{aligned}
$$

Applying Avery-Peterson theorem we obtain the result.

Example 2.1. This example shows a problem that has at least three positive solutions, according to Theorem 3. Suppose that

$$
\begin{gathered}
q(t)=1, \\
f(t, u, v)=\left\{\begin{array}{cc}
t+u^{3}+\left(\frac{v}{55}\right)^{2}, & 0 \leq u \leq 3 \\
t+24+u+\left(\frac{v}{55}\right)^{2}, & 3 \leq u
\end{array}\right.
\end{gathered}
$$

and

$$
M(y)=0.7+0.4 \sin ^{2}(y)
$$

Taking $a=1.5, d=55, A=0.7$ and $B=1.1$ we find 
(A1) for $(t, u, v) \in[0,1] \times[0,55] \times[-55,55]$

$$
\begin{gathered}
r_{1}=\max _{x \in[0,1]} \int_{0}^{1}\left|\partial_{t} G(t, s)\right| d s=\frac{1}{2} \\
0 \leq f(t, u, v) \leq 1+24+55+1=76<77=\frac{55 \times 0.7}{0.5}=\frac{d A}{r_{1}} \\
A \leq M\left(\left\|u^{\prime}\right\|_{2}^{2}\right) \leq B .
\end{gathered}
$$

(A2) for $(t, u, v) \in[0,1] \times[0,1.5] \times[-55,55]$

$$
f(t, u, v) \leq 1+(1.5)^{3}+1=5.375<8.4=\frac{\lambda_{1} a}{r_{2}}
$$

where

$$
r_{2}=\max _{t \in[0,1]} \int_{0}^{1}|G(t, s)| d s=\frac{1}{8} .
$$

(A3) for $(t, u, v) \in[0,1] \times[3,12 \sqrt{2}] \times[-55,55]$

$$
f(t, u, v) \geq 27>26.4=\frac{2 \times 1.5 \times 1.1}{1 / 8}=\frac{2 a B}{r_{3}}
$$

where $r_{3}=\min \left\{\int_{0}^{1} G\left(\frac{1}{4}, t\right) d t, \int_{0}^{1} G\left(\frac{3}{4}, t\right) d t\right\}=\frac{1}{8}$.

Thus, from Theorem 3, the problem has at least three positive solutions.

\section{NUMERICAL SOLUTIONS}

In most studies, numerical solutions are obtained by fixed point methods, according to [12]. More specifically, an iterative sequence based on operator given by equation (2.1) define the method. The basic idea of the proposed method is to use the Levenberg-Maquardt algorithm [13]. This type of algorithm arises frequently in the context of optimization related to the data adjustment problem [7]. The Levenberg-Marquardt algorithm can be easily used for the solution of non-linear systems. Algorithm 1 briefly describes the ideas to solve the Problem (1.1).

The motivation for the Algorithm 1 is the fact that fixed point methods are tendentious to find solutions in which the operator $T$ is a contraction and consequently, chosen an initial approximation $u^{0}$ we have generally two possibilities: the method converges to solution given by Banach's Theorem or the method diverges. Anyway, if we have multiple solutions (as in Theorem 3), we can try to find these solutions. For this reason, our proposed algorithm, using an appropriated initial approximation, allows to find others solutions. So the development of an heuristic to find a better initial approximation is relevant. 


\section{Algorithm 1:}

1. Define an uniformly spaced mesh $\left\{t_{j}\right\}, j=1, \ldots, n$.

2. Choose initial approximation $u_{j}^{0}=u^{0}\left(t_{j}\right)$.

3. Discretize the Problem (1) by finite difference.

For $j=2, \ldots, n-1$

- $u^{\prime \prime k}\left(t_{j}\right)=\frac{u^{k}\left(t_{j+1}\right)-2 * u^{k}\left(t_{j}\right)+u^{k}\left(t_{j-1}\right)}{h^{2}} ;$

- $u^{\prime k}\left(t_{j}\right)=\frac{u^{k}\left(t_{j+1}\right)-u^{k}\left(t_{j-1}\right)}{2 * h}$

approaching $\left\|u^{\prime}\right\|_{2}^{2}$ by using trapezoidal rule.

Thus we have the following linear system

$$
r\left(u^{k}\right)=0,
$$

where

$$
r\left(u^{k}\right)=\left\{M\left(\left\|u^{\prime}\right\|_{2}^{2}\right) u^{\prime \prime k}\left(t_{j}\right)+q\left(t_{j}\right) f\left(t_{j}, u^{k}\left(t_{j}\right), u^{\prime k}\left(t_{j}\right)\right)=0 ; j=2, \ldots, n-1 .\right.
$$

4. For $k=1,2,3, \ldots$ (Levenberg-Maquardt)

(a) Compute $r^{k}=\left(r_{1}, r_{2}, \ldots, r_{n}\right)^{T}$ and $A_{k}=\left(a_{i j}\right)_{n \times n}$;

$$
r_{i}=r_{i}\left(u^{k}\right), a_{i j}=\nabla r_{i}\left(u^{k}\right)
$$

(b) Find $\Delta_{k}$ such that:

$$
\left(A_{k}^{T} A_{k}\right) \Delta^{k}=-A_{k}^{T} r^{k} .
$$

(c) Determines $\alpha_{k}$ such that the Armijo's condition is satisfied.

(d) Compute

$$
u^{k+1}=u^{k}+\alpha_{k} \Delta_{k}
$$

5. Convergence test.

\subsection{An heuristic procedure for initial approximations}

We know the solutions that we are looking for must be concave or convex and will satisfy the condition $u(0)=u(1)=0$. Thus, approaches by parabolas are reasonable ways of approaching the solution. In this sense, the heuristic procedure proposed in this paper consists in generating parables about initial points as follows:

$$
u^{0}(x)=n_{r} x(1-x)
$$


where $n_{r}$ is a random number in $\left[-\frac{d}{2}, \frac{d}{2}\right]$. For practical purposes, the proposed procedure is defined by Algorithm 2.

\section{Algorithm 2:}

1. Choose a vector $n_{r} \in\left[-\frac{d}{2}, \frac{d}{2}\right]^{p}$.

2. For $k=1, \ldots, N$ do:

(a) Compute $u_{k, i}^{0}=u_{k}^{0}\left(x_{i}\right)=n_{r k} x_{i}\left(1-x_{i}\right), i=1, \ldots, n$

(b) Run Algorithm 1 with initial approximation $u_{k}^{0}$.

Naturally, this procedure returns multiple answers. So we need to establish a way to compare solutions in order to distinguish them. Note that the magnitude of the solutions may be different. In this sense, we say that the numerical solutions $u^{*}$ and $u^{* *}$ are equivalent if

$$
\left\|u^{*}-u^{* *}\right\| \leq \max \left\{10^{-8}, 10^{-6} \min \left\{\left\|u^{*}\right\|,\left\|u^{* *}\right\|\right\}\right\} .
$$

is satisfied.

Additional tests are run by using an algorithm based on the Banach Fixed Point Theorem. Considering some hypothesis about the functions $f$ and $M$, we can demonstrate, using the Banach Fixed Point Theorem (details in [12]), the local convergence of algorithms that use the iterative sequence

$$
u^{k+1}=T\left(u^{k}\right)
$$

This procedure is described by Algorithm 3, which is a version of the algorithm proposed in [12].

\section{Algorithm 3:}

1. Define a uniformly spaced mesh $\left\{x_{j}\right\}$ em $[0,1]$.

2. Choose initial approximation $u_{j}^{0}=u^{0}\left(x_{j}\right)$.

3. For $k=1,2,3, \ldots$

(a) Compute $u_{j}^{\prime k}$ by central-differences.

(b) Compute $u_{j}^{k+1}$ using

$$
u^{k+1}=T\left(u^{k}\right)
$$

where the integrals are computed using trapezoidal rule.

4. Convergence test. 
For practical purposes, the procedure is formalized by Algorithm 4, which is equivalent to Algorithm 2 and it calculates possible multiple solutions.

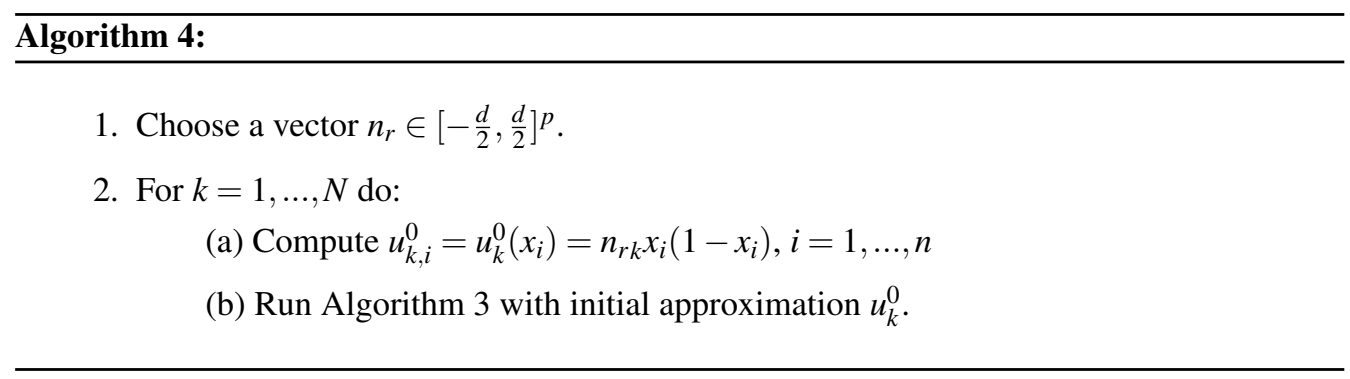

\subsection{Examples}

The examples below show how the Algorithm 2 can be promissor in order to find multiple solutions. We run Algorithm 2 and 4 with $N=10$. For Algorithm 3, we consider as stopping criteria: $\left\|u^{k+1}-u^{k}\right\|<10^{-4}$ and for Algorithm 1 we consider $\left\|u^{k+1}-u^{k}\right\|<10^{-6}$.

Example 3.1. Consider Problem (1.1) defined by

$$
f\left(t, u, u^{\prime}\right)=u
$$

and

$$
M(y)=\frac{1}{\pi^{4}} y+\frac{1}{2 \pi^{2}}
$$

The solutions are $u(x)=\sin (\pi t)$ and $\bar{u}(x)=0$. In this example we consider $d=10$ and $n=20$ points for the spaced mesh. The numerical results are compared with the exact solution.

Running Algorithm 2, the procedure converged to the solution in 9 out of 10 times in which the Algorithm 1 was called. After 7 iterations of Algorithm 1, six initializations converged to the solution $u$ and the obtained accuracy is $\max \left|u^{7}-u\right|=0.006189$. After 4 iterations of Algorithm 1, three initializations converged to the solution $\bar{u}$ and the obtained accuracy is $\max \left|u^{4}-\bar{u}\right|=1.092 \times 10^{-13}$. The divergence means that the maximum number of iterations, $i=50$, was extrapolated. At the point where the execution stopped, the solution was near of $u$.

On the other hand, running Algorithm 4, the procedure converged to the solution $\bar{u}$ (the less norm) in 8 out of 10 times in which the Algorithm 3 was called and reached the required accuracy $\left(\left\|u^{k+1}-u^{k}\right\|<10^{-4}\right)$ after $k$ iterations, with $11 \leq k \leq 14$. The two divergences mean that Algorithm 3 stopped running since the function $M$ became null and then there was a divide by zero. For accuracy or order $<10^{-4}$, the algorithm diverges in most of times, due to divide by zero.

Example 3.2. Consider the Problem (1.1) defined by

$$
f\left(t, u, u^{\prime}\right)=u\left(\pi^{2} u^{2}+u^{\prime 2}\right)
$$


and

$$
M(y)=0.1+\frac{9 \sqrt{2}\left(y^{2}\right)^{\frac{1}{4}}}{10 \pi}
$$

The solutions are $u(x)=\sin (\pi t), \bar{u}(x)=-\sin (\pi t)$ and $\underline{u}(x)=0$. In this example, we consider $d=10$ and $n=20$ points for the spaced mesh. The numerical results are compared with the exacts solutions.

Running Algorithm 2, the procedure converged to the solution in 10 out of 10 times in which the Algorithm 1 was called. After 5 iterations of Algorithm 1, five initializations converged to the solution $u$ and the obtained accuracy is $\max \left|u^{5}-u\right|=0.00532072$. After 5 iterations of Algorithm 1, three initializations converged to the solution $\bar{u}$ and the obtained accuracy is $\max \left|u^{5}-\bar{u}\right|=0.00574833$. After 12 iterations of Algorithm 1, two initializations converged to the solution $\bar{u}$ and the obtained accuracy is $\max \left|u^{12}-\underline{u}\right|=0.000063816$.

On the other hand, running Algorithm 4, the procedure converged to the solution null (the less norm) in 10 out of 10 times in which the Algorithm 3 was called and reached the required accuracy $\left(\left\|u^{k+1}-u^{k}\right\|<10^{-4}\right)$ after $k$ iterations, with $5 \leq k \leq 7$.

We have conducted an additional test, which run Algorithm 2 using the functions defined in Example 1. In this example, we defined $n=20$ and $d=55$. Using the criterion established in (3.1), we obtain that the 3 solutions are different. These results illustrate the result of existence given by Theorem 3. Figure 1 shows a graphical representation of these solutions.

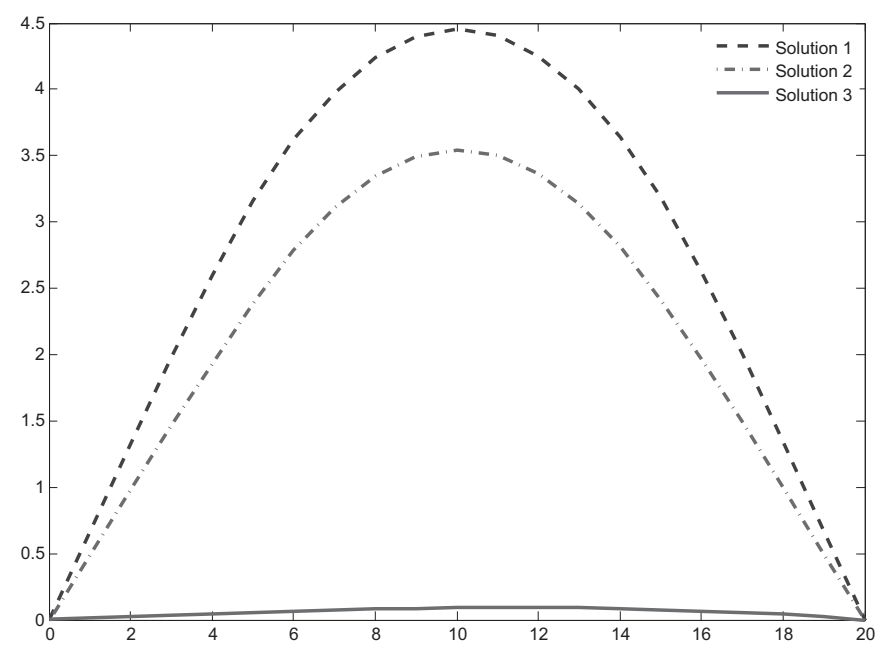

Figure 1: Solutions founded by running Algorithm 2 using Example 1. 
Running Algorithm 4, the procedure converged to the solution of less norm in 9 out of 10 times in which the Algorithm 3 was called (according to the criteria described in 3.1) and reached the required accuracy $\left(\left\|u^{k+1}-u^{k}\right\|<10^{-4}\right)$ after 5 iterations.

As a conclusion, Algorithm 4 have found only the solution of less norm in all the run examples. On the other hand, Algorithm 2 have found, to the same run examples, all the possible solutions. Therefore, Algorithm 2 is more robust and suitable to determine multiple solutions.

\section{FINAL REMARKS}

Considering the numerical aspects of this work, we present a new algorithm (Algorithm 1) and a new heuristic that allows obtain multiple solutions. The method has robustness to solve second order equation Kirchhoff problem. Of course, the cost of this robustness is a slightly higher cost of computer processing, especially when we compare the method presented in last section with fixed-point methods (using the operator given by (7)).

Fortunately, the level of processing is not absurd even considering finer meshes. Furthermore, the method can be adapted for parallel programming and consequently, new features can be exploited in the computational field.

RESUMO. Um problema de valor de fronteira associado é uma equação do tipo Kirchhoff é considerado. Resultados de existência de múltiplas soluções são provados utilizando o teorema de Avery-Peterson. Um método numérico baseado no algoritmo de LevenbergMaquardt combinado com um processo heurístico é apresentado com finalidade de elucidar aspectos numéricos e teóricos.

Palavras-chave: Múltiplas soluções, equação de Kirchhoff, soluções numéricas.

\section{REFERENCES}

[1] C. Alves, F. Corrêa \& T.F. Ma. Positive solutions for a quasilinear elliptic equation of Kirchhoff type. Computers \& Mathematics with Applications, 49(1) (2005), 85-93.

[2] P. Amster \& M.C. Mariani. A fixed point operator for a nonlinear boundary value problem. Journal of mathematical analysis and applications, 266(1) (2002), 160-168.

[3] A. Arosio \& S. Panizzi. On the well-posedness of the Kirchhoff string. Transactions of the American Mathematical Society, 348(1) (1996), 305-330.

[4] R. Avery \& A.C. Peterson. Three positive fixed points of nonlinear operators on ordered Banach spaces. Computers \& Mathematics with Applications, 42(3-5) (2001), 313-322.

[5] G. Caristi, S. Heidarkhani \& A. Salari. Variational Approaches to Kirchhoff-Type Second-Order Impulsive Differential Equations on the Half-Line. Results in Mathematics, 73(1) (2018), 44.

[6] M. Chipot \& J.F. Rodrigues. On a class of nonlocal nonlinear elliptic problems. ESAIM: Mathematical Modelling and Numerical Analysis, 26(3) (1992), 447-467. 
[7] C.T. Kelley. "Iterative methods for optimization", volume 18. Siam (1999).

[8] G.R. Kirchhoff. "Vorlesungen über mathematische physik: mechanik", volume 1. Teubner (1876).

[9] M.I.A. Lourakis. A brief description of the Levenberg-Marquardt algorithm implemented by levmar. Foundation of Research and Technology, 4(1) (2005), 1-6.

[10] T.F. Ma. Existence results for a model of nonlinear beam on elastic bearings. Applied Mathematics Letters, 13(5) (2000), 11-15.

[11] T.F. Ma, E.S. Miranda \& M.B. de Souza Cortes. A nonlinear differential equation involving reflection of the argument. Arch. Math.(Brno), 40(1) (2004), 63-68.

[12] A.L.M. Martinez, E.V. Castelani, J. Da Silva \& W.V.I. Shirabayashi. A note about positive solutions for an equation of Kirchhoff type. Applied Mathematics and Computation, 218(5) (2011), 2082-2090.

[13] J.J. Moré. The Levenberg-Marquardt algorithm: implementation and theory. In "Numerical analysis". Springer (1978), pp. 105-116. 